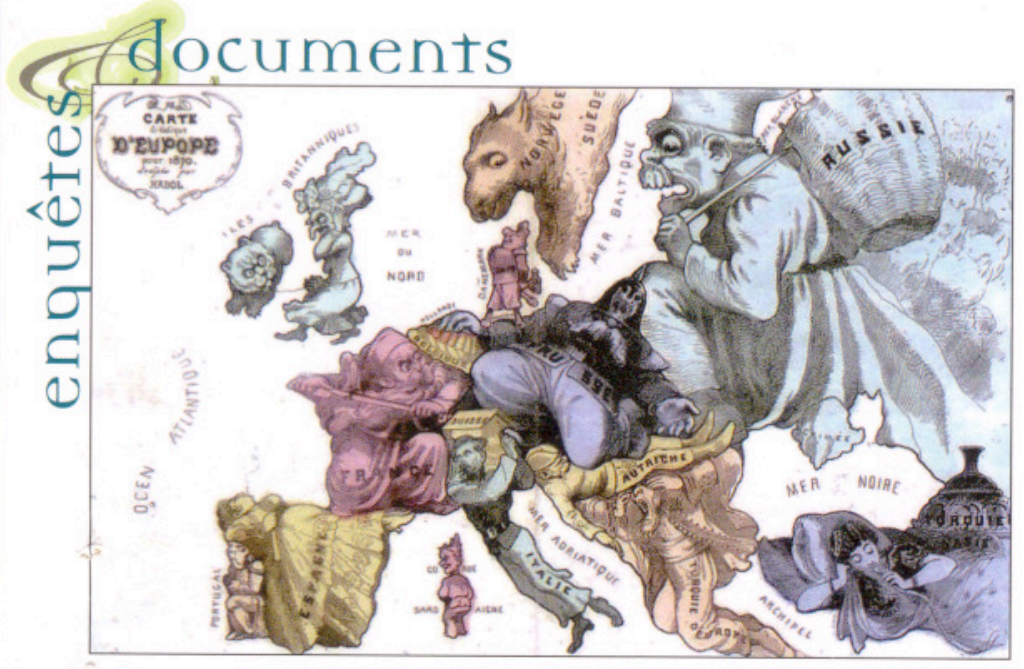

La France

face aux crises et aux conflits des périphéries européennes et atlantiques

du XVII ${ }^{\mathrm{e}}$ au $X X^{\mathrm{e}}$ siècle

Textes réunis par

Éric Schnakenbourg et Frédéric Dessberg 


\section{Alliances de revers et modernisation militaire: le rôle des envoyés militaires français en Europe centrale et orientale, $\mathrm{XVII}{ }^{\mathrm{e}}-\mathrm{XVIII}$ siècles}

La problématique complexe des rapports diplomatiques et militaires entre la France et l'Europe centrale et orientale relève de questions conceptuelles auxquelles il faut répondre afin de déterminer le rôle des coopérations militaires et diplomatiques. Dans notre étude, nous nous proposons d'évoquer les fonctions des agents diplomatiques et militaires afin de souligner l'évolution des missions militaires dans la période concernée. Nous attachons une importance particulière aux conflits internationaux auxquels la politique extérieure française devait réagir dans les régions périphériques. Le terme "périphérie" exige également une définition et explication dans une optique politique et militaire. Nous examinons les problèmes des déplacements et de la transmission des messages dans un espace lointain, ainsi que les méthodes de travail des agents militaires de l'époque. En dernier lieu, nous essayerons de démontrer les effets modernisateurs de l'activité des envoyés militaires français.

Pour réduire le champ d'investigation, nous avons privilégié quelques cas de figure dans le nombre important des envoyés militaires dans le vaste espace de temps couvert par notre période. Premièrement, nous évoquons les envoyés français qui collaboraient avec les révoltés hongrois de la fin du XvII ${ }^{\mathrm{e}}$ siècle et du début du XVIII ${ }^{\mathrm{e}}$ siècle. Parmi ceux qui y travaillaient, il faut souligner l'importance du brigadier général Louis Lemaire dont les souvenirs viennent d'être publiés par les soins du professeur Jean Bérenger ${ }^{1}$. Il a été envoyé en mission en Hongrie au cours de la guerre d'indépendance de François II Rákóczi. Il faisait partie de l'entourage du comte Des Alleurs, lieutenant général choisi par Louis XIV pour diriger une mission mixte à la fois diplomatique et militaire afin de "donner forme de troupes de guerre à ces légions de Mécontents ${ }^{2}$ ". Avec d'autres officiers français, il était placé comme "conseiller technique" auprès du prince François II Rákóczi. Il nous laissa une Relation assez détaillée et très critique de son séjour en Hongrie. L'État du prince François II Rákóczi est doté d'une véritable armée dont la valeur militaire n'est pas négligeable, malgré ses échecs systématiques dans les campagnes. Cependant, la véritable discipline 
n'existe pas dans l'armée kouroutz et les envoyés militaires français s'en plaignent souvent dans leurs rapports. Le brigadier Lemaire nous donne un témoignage sarcastique de valeur militaire d'un des principaux chefs de l'armée kouroutz:

«Forgats n'oubliant pas la tentative de l'ambition de Bercseni, ne perdait pas un moment à le décréditer non seulement dans l'esprit du Prince, mais encore dans celui des autres généraux en lui disant et à eux, qu'il prenait un pouvoir qui obscurcissait tout autre et le Prince même et ne faisait distinguer que sa personne, soit dans les négociations, dans les projets de guerre, dans les expéditions et dans les commandements des troupes dont la direction ne lui convenait pas puisque depuis qu'il s'en mêlait il n'avait établi dans la milice ni règle, ni exercice ou discipline et que de cette manière on ne ferait jamais de bons soldats, ni de bons officiers, il parlait en guerrier consommé et de même que s'il eut été disciple de César ${ }^{3}$."

Le brigadier Lemaire constitue un exemple d'envoyé militaire officiel auprès d'un prince quasiment allié de la France pendant une guerre ouverte avec l'ennemi commun. Un autre cas de figure est fourni par l'activité militaire d'Alexandre de Bonneval ${ }^{4}$ en Turquie qui était plutôt un conseiller militaire de fortune se trouvant en Turquie au moment où la diplomatie française avait besoin de ses services. Militaire chevronné, ayant une expérience internationale reconnue, ce gentilhomme français brouillé avec le prince Eugène de Savoie choisit le turban et le service du sultan. Il a instruit le corps turc qui était confié selon les exercices et les évolutions des armées européennes. Pendant la guerre austro-russoturque de 1737-1739, il contribue à la réforme de l'artillerie turque, en fondant le corps des bombardiers (humbaraçi en turc) et en introduisant une série de réformes dans l'armée ottomane. De concert avec l'ambassadeur de France à Constantinople, le marquis de Villeneuve, il contribue à l'issue heureuse de cette guerre pour la Turquie qui reconquiert la forteresse de Belgrade et conclut un traité de paix avantageux. L'activité remarquable de Bonneval Pacha ouvre une série de missions militaires françaises en Turquie qui continuera jusqu'au $\mathrm{XIX}^{\mathrm{e}}$ siècle. Ce deuxième cas de figure représente donc celui des missions militaires non-officielles, ou secrètes, dans des périodes où la France n'était pas en guerre ouverte.

Le troisième cas de figure est celui du baron de Tott qui est celui d'un expert prédestiné pour sa tâche, et qui réunit les qualités du diplomate et du militaire. François baron de Tott était le second fils d'un émigré hongrois nommé András Tóth, ancien combattant de la guerre d'indépendance hongroise (1703-1711). András Tóth réussit à s'intégrer au sein de l'armée royale française, notamment dans le régiment de hussards Berchény. Grâce à ses connaissances dans la langue turque, il fut même employé dans la diplomatie française. Le marquis de Villeneuve, ambassadeur de la France à Constantinople, constata les qualités linguistiques de Tóth lors d'un de ses séjours de recrutement en Turquie et commença à l'employer pour différentes missions en Orient ${ }^{5}$. Il se distingua en 
particulier durant les négociations de paix de Belgrade terminant la guerre austroturque de 1737-1739, et devint vite un agent expérimenté et fut bientôt initié à la diplomatie secrète de Louis XV, le fameux Secret du Roi. En sa qualité d'agent secret, il fit des démarches en faveur des Hongrois émigrés en Turquie ${ }^{6}$. Pour assurer la continuité des agents hongrois au service de la France en Turquie, le fils cadet d'András Tóth fut également envoyé à Constantinople, en 1755, comme "enfant de langue". Il y passa huit ans et se lia avec l'ambassadeur, le comte de Vergennes, et se maria avec Marie de Rambaud. Il perdit son père en 1757 et une grande partie de ses revenus basés sur les pensions viagères d'András Tóth. Ayant terminé son apprentissage, il retourna en France où il espéra un poste diplomatique digne de sa formation. Une première occasion se présenta en 1766, date à laquelle il fut envoyé à Neuchâtel, principauté prussienne, afin de s'informer sur l'effervescence politique dans la population de cette ville. Sa mission fut rapidement dévoilée, et il dut se retirer en France dès le début de l'année $1767^{7}$.

La véritable possibilité d'un emploi en Orient s'offrit la même année. Le duc de Choiseul envoya François de Tott en Crimée auprès du khan des Tatars en qualité de consul de France en septembre 1767. Il devait inciter le souverain tatar à faire la guerre contre les Russes. Tott camoufla sa vraie mission par des buts secondaires: par exemple par le prétexte de l'achat des chevaux de remonte pour les régiments de hussards en France ${ }^{8}$. Il y réussit à gagner la grâce du khan Kirim Guirây dont il devint le confident. En juillet 1768, des Cosaques brûlèrent la petite ville de Balta en territoire tatar. Cette affaire fournit au baron de Tott l'occasion d'exciter la vengeance du khan et força le Grand Seigneur de lever l'étendard de la guerre. La déclaration en fut faite le 6 octobre 1768. François de Tott accompagna le khan dans une incursion contre les nouveaux établissements russes en Nouvelle Servie. Il assista à la mort de Kirim Guirây auquel il consacra plusieurs pages de ses mémoires ${ }^{9}$. Ayant ainsi rempli sa mission, il se rendit à Constantinople en 1770 .

Dans la capitale ottomane, la situation devint critique après la défaite de la marine turque à Ceshmé le 5 juillet 1770. L'amiral Orloff menaçait déjà d'envahir Constantinople. Ce fût à ce moment-là que le baron de Tott se distingua dans le perfectionnement de la défense turque et contribua à sauver la capitale. Il continua ensuite la réorganisation et la modernisation de l'artillerie turque. Le baron de Tott reprit le projet de Bonneval pacha en utilisant les acquis de la réforme de l'artillerie française moderne représentée par des ingénieurs aussi éminents que Bélidor ou Gribeauval. Il y constitua une école de mathématiques et avec l'aide d'un renégat écossais du nom de Campbell Mustapha Agha et du capitaine d'artillerie français Antoine-Charles Obert (Aubert) qui forma un nouveau corps d'artillerie à tir rapide, nommé des süratçi (sürat topçularî). L'établissement d'une fonderie de canons à Hasköy sous la direction des spécialistes français fut un succès et resta un effet durable de l'activité modernisatrice de Tott. Il devint le favori du jeune sultan Mustapha III et élabora des projets de grande envergure: il envisagea, par exemple, le rétablissement de l'ancien canal 
de Suez. La mort prématurée du sultan mit fin au séjour du baron de Tott en Turquie et il retourna en France en 1776.

En analysant le rôle des envoyés militaires, il faut souligner qu'à l'époque moderne les fonctions des diplomates et des envoyés militaires sont souvent similaires. Même si le caractère des membres du corps diplomatique est à notre époque relativement bien défini, les affaires militaires relèvent souvent du secret et de la confusion avec les tâches diplomatiques. Il est évident que les ambassadeurs et envoyés diplomatiques sont des "honnêtes espions " qui réunissent des renseignements sur le pays de leurs missions, y compris ceux qui intéressent l'état général de l'armée du pays où ils sont envoyés ainsi que les réformes militaires qui s'y produisent durant leur séjour. Les correspondances des ambassadeurs impériaux à Constantinople et à Berlin montrent deux exemples évidents dans les années 1770-1780. Le baron de Thugut remarque tous les événements d'exercices militaires à Constantinople, en général à la fin de ses dépêches ${ }^{10}$. La correspondance du baron Charles Reviczky, ambassadeur impérial à Berlin en 1782 relate surtout les nouvelles détaillées sur les réformes militaires prussiennes ${ }^{11}$. Par ailleurs, les envoyés diplomatiques peuvent avoir des missions de reconnaissance militaire des territoires parcourus pendant leur voyage. Leurs missions secrètes sont évidemment camouflées par d'autres missions (économiques, commerciales, administratives, etc.). Le contraire est beaucoup plus rare et n'existe que dans des cas où les pays respectifs entretiennent des relations amicales. Tel est le cas des agents hongrois envoyés en Turquie pour chercher des recrues et des chevaux pour les régiments de hussards français dans la première partie du XVIII ${ }^{\mathrm{e}}$ siècle $^{12}$. Les ambassadeurs sont souvent entourés d'agents militaires détachés de leurs régiments pour des missions précises. Aux échelons inférieurs de la diplomatie, nous rencontrons fréquemment des militaires chargés d'une mission de consuls ou d'agents diplomatiques en particuliers dans des régions de conflits armés. Ce sont des agents polyvalents qui sont tantôt des négociateurs accrédités, tantôt des conseillers militaires. Les rôles sont interchangeables et complémentaires. Par exemple, le baron de Tott qui est envoyé en Crimée. Le bon négociateur peut se révéler en cas de besoin un conseiller militaire, voire un expert de réforme.

La période concernée, les $\mathrm{XVII}^{\mathrm{e}}$ et $\mathrm{XVII{ } ^ { \mathrm { e } }}$ siècles, est riche en événements militaires, ce sont des siècles belliqueux où les armées puissances européennes s'affrontent avec des moyens de plus en plus sophistiqués. Les luttes de la France contre l'Empire, l'Angleterre, la Prusse ou la Russie poussent à l'adoption d'une politique subversive et à la création des alliances de revers sur les périphéries européennes. La diplomatie française se constitue un réseau d'alliance de petits et moyens états d'Europe centrale et orientale sans compter le redoutable Empire ottoman fort utile lorsque les monarques français sont en conflit militaire avec la maison d'Autriche. Ce système d'alliance comprend essentiellement la Suède, la Pologne, la Transylvanie et les Hongrois mécontents ou malcontents et l'Empire ottoman. Dans la construction des liens entre les différents pays et centres de 
rebelles anti-Habsbourg, les agents français jouent un rôle à la fois important et efficace. La concentration des forces et la réussite des négociations multipartites sont l'œuvre des experts français. Lors de la guerre de Hollande, la diplomatie française, en particulier l'ambassadeur français de Varsovie, le marquis de Béthune, réussit à réunir dans une même alliance les Polonais, les Hongrois révoltés (les kouroutz) et les Transylvains. Le 27 mai 1677, on signe à Varsovie un traité de collaboration entre les alliés français, polonais, transylvains et kouroutz. L'aide française consiste en cent mille tallers (trois cent mille livres tournois environ) de pension annuelle pour les frais d'une armée de quinze mille hommes, et de plus une aide militaire de six mille mercenaires de Pologne ${ }^{13}$. Au mois de septembre, sous la direction des colonels Boham et Forval, un corps de quatre mille hommes, composé surtout des Cosaques recrutés en Ukraine, arrive en Hongrie ${ }^{14}$. Réunis aux forces transylvaines et rebelles, ils écrasent l'armée impériale le 10 octobre 1677 à Nyalábvár. Cette victoire montre bien l'efficacité des experts militaires français dans un territoire lointain. Elle contribue à l'élargissement de la guerre en Hongrie contre les Impériaux sous la direction d'un nouveau chef: Thököly. Le fonctionnement de l'alliance de revers s'avère impeccable dans les années qui viennent, les agents et experts militaires réactivent de concert les rebelles hongrois en cas de besoin pour faciliter la politique des réunions sur les frontières françaises. La réussite de cette coopération franco-hongroise est évoquée ainsi, un siècle après les événements, par CharlesEmmanuel de Warnery: "Avant la paix de Riswick la France avait un très puissant allié dans son bon ami le Turc, et les Hongrois de Tökeli n'étaient pas à regarder pour rien. Cette guerre de Hongrie donnait seule assez d'occupation aux troupes de l'empereur, le Nord n'était de même point tranquille, ainsi la France sans alliés ne faisait pas seule la guerre à toute l'Europe ${ }^{15}$."

Pendant la guerre de Succession d'Espagne, l'alliance de revers resurgit en Hongrie avec la guerre d'indépendance de François II Rákóczi. Louis XIV profite de cette opportunité, malgré ses réticences envers les Hongrois qui se sont révoltés contre leur roi légitime. L'alliance de revers reste ainsi une alliance informelle, mais le roi français envoie en Hongrie un représentant, le comte des Alleurs et plusieurs experts militaires, dont le plus connu est le brigadier Lemaire. Grâce aux mémoires de ce dernier, nous connaissons les difficultés des envoyés militaires occidentaux dans une armée traditionnelle et quasiment féodale. L'activité de des Alleurs et de Lemaire prouve encore une fois le caractère complémentaire des diplomates et des militaires: le premier donne souvent des conseils militaires au prince Rákóczi, tandis que le second sert plutôt d'expert militaire aux officiers de l'armée du prince. Malgré les conseils des deux envoyés français, les Hongrois perdent toutes les batailles, mais se révèlent excellents partisans dans les opérations de petite guerre. Après la fin de la guerre hongroise, nombreux sont les anciens combattants qui quittent leur pays et entrent dans l'armée royale française pour y former des célèbres régiments de hussards. Parmi ceux-ci les meilleurs sont employés à leur tour en Europe 
orientale, notamment en Turquie et en Crimée, comme agents ou experts militaires au service de la politique extérieure française. Les projets du Secret du Roi en Europe orientale et l'ouverture de la question d'Orient vers la fin du siècle favorisent leur emploi dans la périphérie de l'Europe.

Après ce rapide survol, il est tout à fait légitime de s'interroger sur la signification du mot "périphérie». L'emploi de ce mot suppose qu'il existe aussi un "centre", une entité géographique qui correspond dans notre cas à l'Europe occidentale. L'idée de l'opposition "centre-périphérie" existe dans la pensée de Fernand Braudel et cette théorie a été exploitée par d'autres historiens, notamment l'américain Immanuel Wallerstein ${ }^{16}$ qui considéraient surtout les caractéristiques économiques des régions européennes. Le centre marqué par un développement commercial et industriel devient à l'époque moderne le berceau du capitalisme, tandis que les périphéries sombrent dans une économie basée sur la production agricole. L'évolution sociale reflète ces différences : à l'ouest, la bourgeoisie s'enrichit, tandis qu'à l'est la société féodale demeure (système du second servage $=$ zweite Leibeigenschaft). Cette théorie a été nuancée par les chercheurs d'Europe centrale, comme Jenö Szücs, qui élabore, dans son ouvrage intitulé Les trois Europes, l'idée d'une zone intermédiaire, une Europe centrale des états moyens comme la Pologne, la Bohême et la Hongrie qui constituent des pays semi-périphériques, surtout par rapport à la Russie et l'Empire ottoman ${ }^{17}$. Ces théories sont bien utiles pour faire des cours d'histoire, mais, en réalité, ne reflètent pas la situation des rapports des forces. En histoire militaire, notamment, l'idée du centre et des périphéries est plus difficile à cerner. Comment peut-on admettre que l'armée espagnole, les fameux tercios du début du XVII ${ }^{e}$ siècle, sont des forces périphériques? Où bien qui pourrait qualifier l'armée russe de Catherine II d'importance secondaire? En plus, les périphéries européennes sont parsemées de centres d'importance stratégiques liés à l'existence des puissances périphériques. Il en résulte une hiérarchie des alliances et des politiques à suivre. Dans l'optique de la diplomatie française, les Malcontents hongrois ou les Tatars de Crimée dépendent de l'appui de l'Empire ottoman et de la Pologne, qui sont des deux centres régionaux. La carrière du comte Des Alleurs montre bien l'importance des lieux: d'abord envoyé auprès du prince Rákóczi, ensuite il devient ambassadeur de France à Constantinople ${ }^{18}$.

En histoire militaire, nous avons un concept qui correspond aux questions du développement militaire à l'époque moderne: c'est la théorie de la fameuse "révolution militaire" développée par des historiens anglo-saxons comme Michael Roberts ou Geoffrey Parker ${ }^{19}$. Cette théorie décrit schématiquement les changements dans l'organisation, dans la tactique et surtout dans la technologie militaire. Ces changements se déroulent dans les forces militaires des grandes puissances, alors que les États petits et moyens n'y arrivent que grâce à une assistance extérieure. L'aide peut consister en armes, en argent et surtout en envoyant des missions militaires dans les pays ciblés. Tel est le cas des envoyés militaires en Hongrie ou en Pologne au tournant des XVII ${ }^{e}$ et XVIII ${ }^{e}$ siècles. 
La révolution militaire n'a pas marqué d'une manière égale tout le territoire de l'Europe. Dans son ouvrage magistral, Lucien Bély parle des "résistances à la révolution militaire" dans les périphéries européennes. Il s'agit essentiellement de l'Europe centrale et orientale où il y avait peu de défenses bastionnées, et la cavalerie, surtout la cavalerie légère, dépasse largement l'infanterie dans la seconde moitié du XVII ${ }^{\mathrm{e}}$ siècle ${ }^{20}$. Cette résistance s'avère même efficace et favorise, au cours du xvIII ${ }^{e}$ siècle, le succès de la tactique de la petite guerre dans les armées occidentales. Néanmoins, ces quelques exceptions ne font que confirmer les règles du processus de la révolution militaire. À l'époque des Lumières, certaines puissances "périphériques " reconnaissent leur retard dans le domaine militaire et recrutent des experts et réalisent des réformes militaires toujours dans la lignée de la fameuse "révolution militaire». Le recrutement des experts militaires étrangers vise à moderniser la structure entière des armées, tandis que les missions militaires éphémères ne sont souvent que des remèdes d'urgence pour éviter le pire. En tout état de cause, le développement militaire est une condition sine qua non de l'existence d'une puissance. Le concept du despotisme éclairé reflète bien l'ambiguïté des réformes de certaines puissances de la "périphérie" dans la perspective de rattraper leur retard militaire, bien souvent avec succès comme l'exemple de Russie de Catherine II l'illustre bien. En revanche, la théorie du despotisme oriental qui a provoqué un grand débat au $\mathrm{XVIII}$ siècle, démontre bien que le manque de réformes suffisamment profondes ne permet aux envoyés militaires que d'emporter des succès momentanés et précaires.

Comment travaille un envoyé militaire? Malgré les histoires rocambolesques des envoyés aventuriers, ils opèrent rarement seuls. Derrière les envoyés renommés, il y a souvent une équipe d'experts militaires recrutés sur le terrain ou détachés d'autres services diplomatiques ou militaires. Par exemple, le brigadier Lemaire est entouré de plusieurs autres officiers français qui étaient enrôlés dans les "troupes étrangères" de l'armée du prince Rákóczi. Alexandre de Bonneval crée un véritable réseau d'officiers militaires, parmi eux nous trouvons des déserteurs autrichiens, des réfugiés hongrois (André de Tott), des renégats comme l'illustre Ibrahim Mütteferrika. Le baron de Tott trouve à Constantinople des officiers auxiliaires, qu'il oublie ingratement en écrivant ses mémoires quand il raconte qu'il n'avait sous la main que les exemplaires illustrés de l'Encyclopédie de Diderot et de d'Alembert pour réaliser ses canons à tir rapide. Or, les documents d'archives montrent bien qu'il y a plusieurs experts dans son entourage, comme l'artilleur Obert et un renégat écossais nommé Campbell Mustapha Aga. La vanité est un facteur important dans la formation de la légende qui entoure l'activité des envoyés militaires.

La méthode de travail de l'envoyé est totalement différente de celle d'un officier subordonné dans une armée européenne. Il est plus autonome, plus libre, mais il doit affronter des difficultés de toutes parts. L'invention et l'intuition jouent un rôle primordial et ses initiatives doivent répondre aux possibilités et 
aux besoins du terrain. Ses ordres arrivent de loin ce qui lui confère une large autonomie d'action. Néanmoins, il doit agir de concert avec les autorités militaires locales ce qui lui pose le plus de problèmes quotidiens. Le brigadier Lemaire n'arrive jamais à vaincre la méfiance et l'animosité des officiers supérieurs hongrois. Bonneval doit se convertir à l'islam pour se faire accepter par les Turcs. Le baron de Tott, dans ses Mémoires, fustige le fanatisme et la superstition des autorités turques auxquelles il attribue les échecs de la guerre russo-turque de 1768-1774. Le célèbre historien américain, Theodore Orvell Murphey dans sa monographie consacrée à la carrière de Vergennes, Charles Gravier comte de Vergennes, French Diplomacy in the Age of Revolution 1719-1787, remarque avec justesse le caractère ambivalent des envoyés. Il considère les deux officiers de hussards attachés au service de l'ambassadeur français, André de Tott et son fils François, comme les prototypes des conseillers militaires d'aujourd'hui qui ne savent pas toujours envers qui ils sont véritablement responsables ${ }^{21}$.

Un envoyé militaire doit s'adapter aux règles du terrain. Dans un premier temps, il ne s'agit pas forcément de transformer l'art militaire traditionnel du pays en méthodes de guerre occidentales, mais de réaliser des opérations militaires ponctuelles avec une concentration optimale des forces existantes. C'est pourquoi le colonel Boham recrute plutôt des Cosaques, cavaliers légers d'Europe orientale, pour lutter contre les Impériaux en 1677. Le comte des Alleurs conseille au prince Rákóczi d'employer la guerre à la hussarde, la petite guerre, contre les Autrichiens et d'éviter les batailles rangées. Ces exemples vont à l'encontre des théories figées de l'historiographie militaire qui décrivent la relation entre les grandes et petites guerres dans une perspective géographique est-ouest ${ }^{22}$.

Les domaines dans lesquels les envoyés militaires agissent sont très variés. En général, il s'agit d'un travail d'ingénieur compliqué où la formation des officiers occidentaux se montre absolument supérieure à celle de leurs homologues du pays d'accueil. Par exemple, pour les représentations cartographiques, les officiers français s'étonnent souvent des lacunes qu'ils constatent dans les pays lointains, comme le baron de Tott le remarque sarcastiquement dans ses mémoires: "Aucun vaisseau n'avait encore paru à Constantinople: donc les Russes n'ont pas de vaisseaux, ou si par hasard ils en ont, cela ne fait rien aux Turcs, puisqu'il n'y a point de communication entre la Baltique et l'Archipel ${ }^{23}$." Le comte Alexandre de Bonneval fournit des cartes militaires aux Turcs qu'il fait même imprimer par un renégat d'origine transylvaine, Ibrahim Müteferrika, qui a fondé la première imprimerie dans l'Empire ottoman. Le baron de Tott prépare aussi des cartes militaires pour la direction de l'armée ottomane pendant la guerre russo-turque de 1768-1774.

L'autre domaine important de l'activité des envoyés militaires en territoire ottoman est celui de l'artillerie. Malgré les progrès rapides des armées turques dans cette arme, il faut rappeler que les succès les plus spectaculaires (la prise de Constantinople en 1453 ou la bataille de Mohács en 1526) sont dus aux artilleurs renégats, le plus souvent des Italiens. Cette arme savante représente 
un des piliers les plus remarquables du processus de modernisation des armées occidentales européennes de l'époque moderne. Les historiens qui étudient les questions de modernisation de l'armée ottomane à cette époque y attachent beaucoup de d'importance ${ }^{24}$. Dans notre étude, nous soulignons en particulier les travaux d'Alexandre de Bonneval et de François de Tott dans l'introduction des nouvelles technologies de l'artillerie dans l'armée ottomane.

Si nous évoquons les armes savantes, il est indispensable de parler des fortifications qui, parallèlement aux progrès de l'artillerie, connaissent un développement spectaculaire en Europe occidentale, mais que les régions périphériques suivent souvent avec un retard considérable, comme nous pouvons le remarquer dans le cas de la Pologne ou la Hongrie. La situation de l'Empire ottoman reste particulière. Tandis que ses confins sont renforcés par des forteresses stratégiques puissantes et modernes, ses régions centrales sont dans une situation très différente. Le marquis de Silva remarque dans son ouvrage la faiblesse des Turcs dans l'art de la fortification: "Leurs ingénieurs ne sont pas plus habiles que les artilleurs. Les fortifications sont ou mauvaises, ou dans l'état le plus dégradé. Les nouvelles n'ont ni flancs ni ouvrages bien construits \& les anciennes ne sont point entretenues. Les Bassas, qui ne songent qu'à amasser de l'argent, les laissent dépérir \& n'ont garde d'y faire des réparations. Il n'y a pas dans tout l'Empire une seule place en état de soutenir un long siège ${ }^{25}$. "L'activité du baron de Tott dans la fortification des Dardanelles et du Bosphore reste un moment symbolique de la réussite des envoyés militaires européens dans la fortification. Et nous pourrions y ajouter des questions diverses concernant le génie militaire de l'époque, comme les questions techniques du transport militaire, qui trouvent leur place dans le travail des officiers européens et dans leurs missions militaires en Europe centrale et orientale.

Une autre branche des réformes introduites par les envoyés militaires vise à opérer des changements plus profonds et rencontre de ce fait des oppositions plus virulentes de la part de l'élite militaire des armées traditionnelles. La discipline militaire massivement introduite en Europe occidentale, a peu de sens dans l'armée des rebelles hongrois du prince François II Rákóczi caractérisée par l'art de la petite guerre et l'idéologie de la liberté nobiliaire qui s'oppose diamétralement à toute idée de subordination autre que celle établie par l'ordre social. La discipline rencontre des obstacles religieux dans l'Empire ottoman, comme le célèbre passage des Mémoires du baron de Tott le montre: "Cette multitude d'imbéciles fanatiques osaient même reprocher aux Russes quelques attaques que ceux-ci avaient fait pendant le saint temps du Ramazan ${ }^{26}$. " Un autre volet des réformes structurelles porte sur la formation pratique et théorique des militaires. Dans cette catégorie, nous pouvons souligner les exemples de la constitution des corps des humbaraçi et süratçi nés des changements introduits par le comte de Bonneval et le baron de Tott. Le sommet des réformes théoriques est la fondation d'une école de mathématiques par le baron de Tott pour la formation des militaires des armes savantes et de la marine. Le succès 
de ces réalisations est confirmé par les missions ultérieures, comme celle de Lafitte-Clavé dans les années 1780, dont les rapports précis démontrent clairement le besoin d'avoir des envoyés militaires en Turquie ${ }^{27}$.

En guise de conclusion, nous pouvons constater à travers ces quelques exemples une transformation du rôle des envoyés militaires en Europe centrale et orientale. Dans un premier temps, il s'agit d'interventions ponctuelles pour réactiver le système d'alliance de revers en cas de besoin, mais volonté de réformer le système militaire des alliés de la France. L'action des envoyés est alors strictement subordonnée aux fins politiques qui déterminent les missions, comme le cas des missions du comte des Alleurs et du brigadier Lemaire nous le montre bien pendant la guerre d'indépendance hongroise. Il y a néanmoins des exceptions qui, tout en confirmant la règle, font avancer les choses dans une autre direction. Il s'agit avant tout d'aventuriers, de transfuges, d'émigrés qui profitent de leur expérience au sein des armées internationales de monarques occidentaux pour passer au service des souverains orientaux, contribuant ainsi largement à une diffusion des technologies militaires. La libre circulation des experts militaires favorise ce phénomène à une échelle remarquable. Ainsi, nous assistons, vers la fin du XVIII ${ }^{\mathrm{e}}$ siècle, à la transformation des missions ponctuelles en une véritable coopération militaire avec les alliés potentiels de la France dans l'espace périphérique européen en vue de réformes de plus en plus profondes et structurelles.

Ferenc TóTH Professeur d'Histoire moderne, université de Szombathely (Hongrie)

\section{NOTES}

1. Brigadier général Louis LE MAIRE, Relation abrégée de ce qui s'est passé dans la guerre de Hongrie depuis le commencement de la campagne de 1705 jusqu'au mois de mars 1708, Jean BéRENGER (éd.), Paris, Honoré Champion, 2007.

2. Jean BÉRENGER, "Un exemple de coopération militaire franco-hongroise: la mission du brigadier général Le Maire pendant la guerre d'indépendance de François II Rákóczi n, Zita TringL et Ferenc Tотн (dir.), Mille ans de contacts II, Relations franco-hongroises de l'an mil à nos jours, Szombathely, 2004, p. 41-42.

3. Brigadier-général Louis Le MAIRE, Relation abrégée..., op. cit., p. 188-189.

4. Sur ce personnage, voir Heinrich BENEDIKT, Der Pascha-Graf Alexander von Bonneval 1657-1747, Graz-Köln, 1959, et Albert VANDAL, Le pacha Bonneval, Paris, 1885.

5. Albert VANDAL, Une ambassade française en Orient sous Louis XV, La mission du marquis de Villeneuve 1728-1741, Paris, 1887, p. 197.

6. Centre des Archives Diplomatiques de Nantes (dorénavant CADN), série Saint-Priest 158.

7. Georges LivET, Recueil des instructions données aux ambassadeurs et ministres de France, tome XXX, Suisse, vol. II, Paris, CNRS éditions, 1983, p. 807-811.

8. CADN, série Saint-Priest 207, pièce n ${ }^{\circ} 5$.

9. Ferenc TÓTH (éd.), Mémoires du baron de Tott sur les Turcs et les Tartares, Maestricht, 1785, Paris, Honoré Champion, 2004. 
10. Haus-, Hof- und Staatsarchiv (Vienne, désormais HHuStA), Türkei II 57 (Berichte 1771) série Turcica 1771.

11. HHuStA, Preussen Kt. 62 Korrespondenz 1782-1783.

12. Voir à ce sujet Ferenc TóTH, "Agents hongrois au service de la France dans la première moitié du XVIII ${ }^{\mathrm{e}}$ siècle", dans Marie PAYET et Ferenc TOTH (dir.), Mille ans de contacts, Relations francohongroises de l'an mil à nos jours, Études françaises de Szombathely II, Szombathely, 2001, p. 5, et $47-59$.

13. Zsolt TrócsánY, Teleki Mihály, Erdély és a kurucmozgalom 1690-ig (Teleki Mihály, La Transylvanie et le mouvement kouroutz jusqu'en 1690), Budapest, 1972, p. 187-204.

14. Ioan HUDITA, Répertoire des documents concernant les négociations diplomatiques entre la France et la Transylvanie au XVIF siècle (1636-1683), Paris, 1926, p. 168.

15. Charles-Emmanuel de WARNERY, Commentaires sur les commentaires du comte de Turpin sur Montecuculi, avec des anecdotes relatives à l'histoire militaire du siècle présent et des remarques sur Guibert et autres écrivains anciens et modernes, tome I, St. Marino, 1777, p. 222.

16. Voir à ce sujet Immanuel WallersteIn, Capitalisme et économie-monde, 1450-1640, Paris, 1980.

17. Jeno Szücs, Les trois Europes, Paris, L'Harmattan, 1992.

18. Voir sur la carrière de Pierre Des Alleurs: Pierre Duparc (éd.), Recueil des instructions données aux ambassadeurs et ministres de France depuis les traités de Westphalie jusqu'à la Révolution française, tome XXIX: Turquie, Paris, CNRS éditions, 1969,p. 187-189.

19. Geoffrey PARKER, La révolution militaire, La guerre et l'essor de l'Occident 1500-1800, Paris, Gallimard, 1993.

20. Lucien Bély, Les relations internationales en Europe XVIF -XVII siècles, Paris, PUF, 1992, p. 319-320.

21. Orville Theodore MurPHY, Charles Gravier Comte de Vergennes, French Diplomacy in the Age of Revolution: 1719-1787, Albany, State University of New York Press, 1982. p. 80.

22. Hervé Coutau-Bégarie, Traité de stratégie (5e éd.), Paris, Economica, 2006, p. 238-243.

23. Cité par Ferenc То́тн, La guerre russo-turque (1768-1774) et la défense des Dardanelles. L'extraordinaire mission du baron de Tott, Paris, Economica, 2008, p. 61.

24. Nous pensons ici surtout aux travaux de Virginia Aksan et de Gábor Ágoston.

25. Marquis de SILVA, Pensées sur la tactique, et la stratégique ou vrais principes de la science militaire, Turin, 1778 , p. 25.

26. F. TÓTH (éd.), Mémoires du baron de Tott..., op. cit., p. 244.

27. Jean BÉrENGER, "Les vicissitudes de l'alliance militaire franco-turque (1520-1800)", Daniel TOLLET (dir.), Guerres et paix en Europe centrale, Mélanges d'histoire des relations internationales offerts à Jean Bérenger, Paris, PUPS, 2003, p. 323-327. 


\section{$\mathrm{e}^{\mathrm{d}}$ \\ La France face aux crises et aux conflits des périphéries européennes et atlantiques}

Ce volume rassemble les contributions qui ont été présentées lors de deux journées d'études organisées en 2008 et 2009 par le Centre de Recherche en Histoire Internationale et Atlantique de l'université de Nantes (CRHIA) et les Écoles militaires de Saint-Cyr Coëtquidan. Les intervenants étaient invités à réfléchir à la notion de périphérie dans la politique étrangère de la France du XVII ${ }^{e}$ au XXe siècle. L'objectif général est de savoir si et comment, sur cette longue période, il est possible de caractériser une permanence dans les objectifs diplomatiques et dans les pratiques militaires de la France, tels qu'ils se révèlent au miroir des conflits et des crises des régions situées au-delà de son voisinage. Les régions continentales privilégiées étaient celles de la grande dorsale allant de la mer Baltique à la mer Noire, ainsi que l'espace maritime comprenant la Méditerranée occidentale et l'Atlantique.

Il a fallu s'interroger sur la notion même de périphérie européenne pour en montrer le caractère dynamique et relatif. Elle doit avant tout être entendue comme un espace intégré à la politique étrangère de la France. Si l'action politique et militaire dans les périphéries n'est pas la priorité des gouvernements français, elle n’en participe pas moins au succès général de son action extérieure. L'interrogation sous-jacente porte sur la capacité de la France à peser, par la diplomatie ou l'action militaire, sur les crises et les conflits de régions plus ou moins lointaines qui demandent une mobilisation de moyens pour connaître et comprendre les situations locales; pour pacifier ou, au contraire, activer les conflits; pour conserver ou modifier les équilibres des forces régionales par la diplomatie ou l’intervention armée.

Éric SCHNAKENBOURG, maître de conférences d'Histoire moderne à l'université de Nantes est membre de l'Institut Universitaire de France. Ses travaux portent sur l'histoire de la politique étrangère française à l'époque moderne, en particulier dans ses rapports avec les pays du Nord. Il a notamment publié La France, le Nord et l'Europe au début du XVIII ${ }^{e}$ siècle, Honoré Champion, 2008.

Frédéric DESSBERG, agrégé et docteur en histoire. Maître de conférences à l'université Paris I-Panthéon Sorbonne, détaché aux écoles de Saint-Cyr Coëtquidan. A publié Le triangle impossible. Les relations franco-soviétiques et le facteur polonais dans les questions de sécurité en Europe (1924-1935), Bruxelles, PIE-Peter Lang, 2009.

\section{En couverture: Carte drolatique d'Europe pour 1870, par Madol.}

Avec le soutien de l'université de Nantes et du CREC Saint-Cyr Coëtquidan.

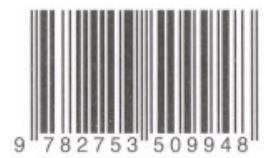

